Accepted author's manuscript. Published in final edited form as: BMJ Quality and Safety 2017; 26(10): 799-805. Publisher DOI: $10.1136 /$ bmjas-2016-006239

\title{
Simplification of the HOSPITAL score for predicting 30-day readmissions
}

Running title: Simplified HOSPITAL score and readmissions.

Word count: 2754

Number of tables: 3

Number of appendix: 0

Manuscript category: Original Research.
Abstract word count: 275

Number of figures: $\mathbf{2}$

Number of references: 30 


\section{ABSTRACT}

\section{Objective}

The HOSPITAL score has been widely validated and accurately identifies high-risk patients who may mostly benefit from transition care interventions. Although this score is easy to use, it has the potential to be simplified without impacting its performance. We aimed to validate a simplified version of the HOSPITAL score for predicting patients likely to be readmitted.

\section{Design and setting}

Retrospective study in 9 large hospitals across 4 countries, from January through December 2011.

\section{Participants}

We included all consecutively discharged medical patients. We excluded patients who died before discharge or were transferred to another acute care facility.

\section{Measurements}

The primary outcome was any 30-day potentially avoidable readmission. We simplified the score as follow: 1) "discharge from an oncology division" was replaced by "cancer diagnosis or discharge from an oncology division"; 2) "any procedure" was left out; 3) patients were categorized into 2 risk groups (unlikely and likely to be readmitted). The performance of the simplified HOSPITAL score was evaluated according to its overall accuracy, its discriminatory power, and its calibration.

\section{Results}

Thirty-day potentially avoidable readmission rate was $9.7 \%(n=11,307 / 117,065$ patients discharged). Median of the simplified HOSPITAL score was 3 points (IQR 2-5). Overall accuracy was very good with a Brier score of 0.08 and discriminatory power remained good with a C- 
statistic of 0.69 (95\% $\mathrm{Cl} 0.68-0.69)$. The calibration was excellent when comparing the expected with the observed risk in the 2 risk categories.

\section{Conclusion}

The simplified HOSPITAL score has good performance for predicting 30-day readmission.

Prognostic accuracy was similar to the original version, while its use is even easier. This simplified score may provide a good alternative to the original score depending on the setting.

Keywords: Patient readmission, score, risk factor, transition of care. 


\section{INTRODUCTION}

Hospital readmissions are common, detrimental for patients, and associated with significant costs for the healthcare system. ${ }^{12}$ In the United States in 2011-2014, more than $15 \%$ of Medicare beneficiaries aged 65 years or older were readmitted within 30 days of discharge after a medical hospitalization. ${ }^{3}$ Preventing readmissions is therefore an important goal for the patients who would benefit from a reduction in the burden of hospitalization, including the risks associated with each new hospitalization, as well as for the healthcare system that would benefit from a reduction in the healthcare costs. It remains however still a challenge to prevents these undesirable events. Although it is estimated that about $73 \%$ of readmissions are not preventable, some of them may still be avoidable. ${ }^{4}{ }^{5} \mathrm{~A}$ recent review including 42 trials on preventive interventions showed that readmission rate could be significantly reduced, with a pooled risk ratio of $0.82\left(95 \%\right.$ confidence interval $[\mathrm{Cl}]$ 0.73-0.91). ${ }^{6}$ However, the most effective interventions were also the most complex and intensive ones, addressing multiple factors related to patient context and capacity, and including among others functional status, caregiver capabilities, socioeconomic factors, or potential for self-management. ${ }^{6}$ Because of the complexity and costs associated with such interventions, hospital physicians need to target them on the group of patients who are most likely to benefit, which might be patients who are at high risk of experiencing a readmission in the absence of any intervention. Unfortunately, clinicians and nurses are not good at identifying which patients are at high risk of readmission. They are actually not doing better than chance alone (C-statistic 0.50 to 0.58$){ }^{7}$ Prediction models may help to better identify those high-risk patients.

The HOSPITAL score is a simple predictor model using 7 clinical variables at discharge (Table 1). It has been validated among nearly 200,000 patients in 5 different countries and showed overall 
good performance to predict the risk of readmission. ${ }^{2-12}$ Although most of its components are easily available, some variables of the original score would be removed to further simplify its use in real-life, and the scoring system would be simplified.

In this study, we aimed to develop and internally validate a simplified version of the HOSPITAL score, which would be easier to uptake for clinicians.

Table 1. Original and simplified HOSPITAL score for 30-day potentially avoidable readmissions.

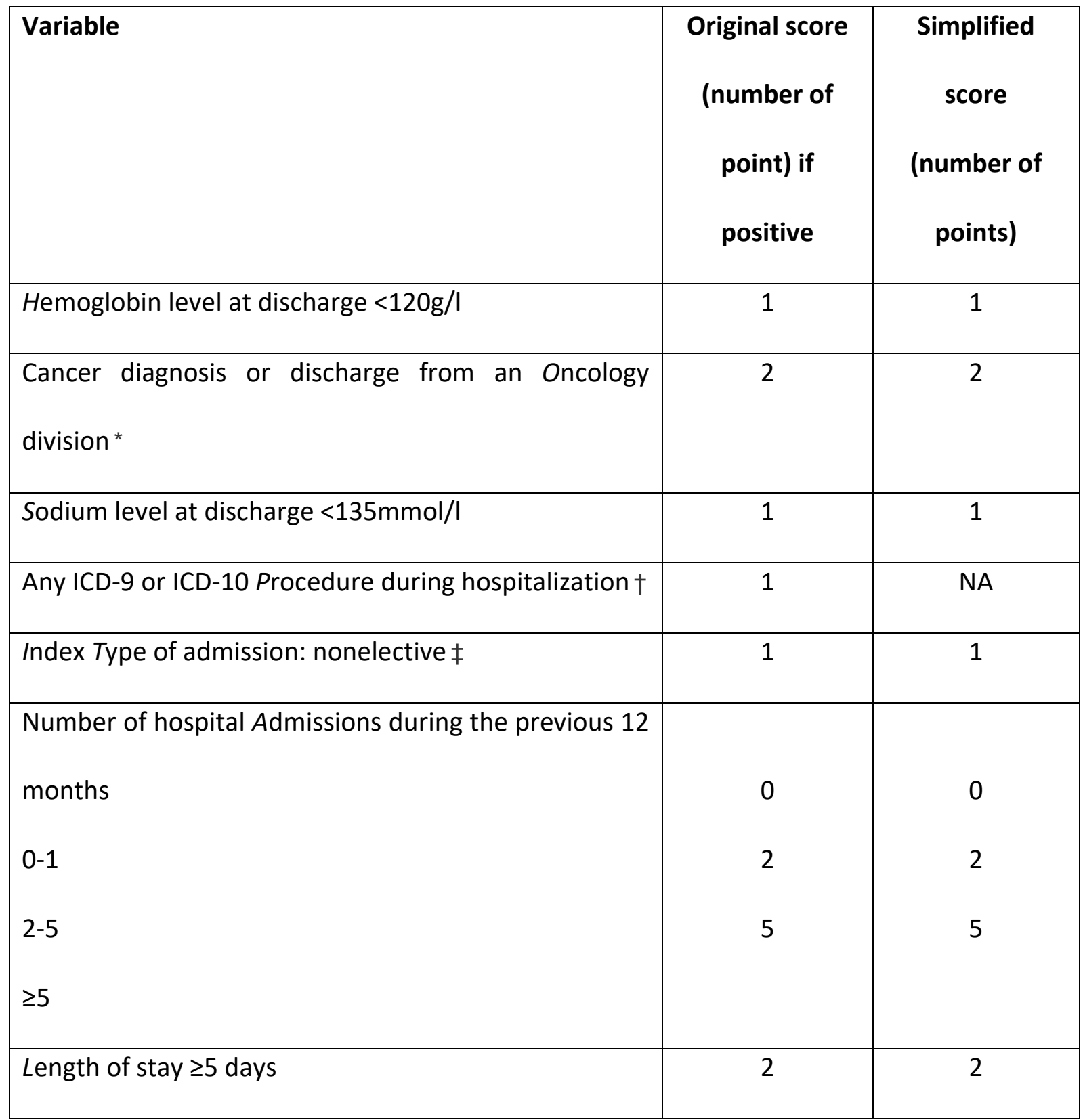




\begin{tabular}{|l|c|c|}
\hline Total & 13 & 12 \\
\hline
\end{tabular}

Abbreviations: ICD, International Coded of Diseases.

* "Discharge from an Oncology division" in the original version of the score.

$\dagger$ This variable was left out in the simplified version of the score.

$\ddagger$ defined as not scheduled in advance for treatment or investigation.

\section{MATERIAL AND METHODS}

\section{Study Design and Setting}

We used the International Cohort of Avoidable REadmissions (ICARE), which is a retrospective cohort of 7 university and 2 community hospitals in United States, Canada, Switzerland, and Israel. Details on the participating hospitals, which were all not-for-profit centers, have been described in detail elsewhere. ${ }^{2}$

The study followed the criteria from the "Transparent reporting of a multivariable prediction model for individual prognosis or diagnosis" (TRIPOD) Initiative. ${ }^{13}$ The managing site (Brigham and Women's Hospital/Partners Healthcare, Boston, Massachusetts) and the institutional review board of each local hospital approved the trial protocol.

\section{Data Source and Participants}

The ICARE included all consecutive medical patients aged $\geq 18$ years and discharged from January $1^{\text {st }}, 2011$, through December $31^{\text {st }}, 2011$ at each participating hospital. Exclusion criteria were: 1) death before discharge; 2 ) transfer to another acute somatic or psychiatric hospital; 3) observation stay and/or length of stay of 1 day or less; 4) discharge against medical advice.

\section{Predictor Variables}


HOSPITAL is the acronym for the 7 predictor variables included in the original derived model (Table 1): ${ }^{14}$ Hemoglobin before discharge (positive if $<120 \mathrm{~g} / \mathrm{l}$ ), discharge from an Oncology division, Sodium level before discharge (positive if $<135 \mathrm{mmol} / \mathrm{I}$ ), any ICD-915 or ICD-10 16 coded Procedure during index hospitalization, Index Type of admission (positive if nonelective, i.e. not scheduled in advance for treatment or investigation), number of hospital Admissions within the 12 months before index admission, and Length of hospital stay (positive if at least 5 days). The same definition as in the derivation study was used to collect all variables at each site. ${ }^{14}$ As all patients from 2 sites had been admitted at least once within the previous 12 months, we imputed 0 point instead of 1 to all patients with a single admission within this time frame. Missing values for hemoglobin $(n=6,907,5.9 \%)$ or sodium $(n=3,980,3.4 \%)$ were considered as normal and therefore attributed 0 point.

Two elements of the score were modified in this study. First, the variable "discharge from an oncology division" was replaced by "discharge from an oncology division or any active cancer diagnosis", because many hospitals don't have their cancer patients in a specific oncology division as faced in a prospective validation study of the score, ${ }^{8}$ and because the cancer diagnosis was as highly associated with 30 -readmission as oncology division. ${ }^{14}$ The diagnosis of cancer was based on the following ICD-9 codes: C00 to C96, C7A, C7B, D00 to D49. Second, "any procedure during index admission" was left out, since this variable could on the one hand be less easily collected, and on the other hand was always the least significant of the model in the validation studies. ${ }^{214}$ Our assumption was that the score would be even more simple to calculate without this variable, and that the performance of the score would remain good. All patients were imputed 0 point for the variable that was left out, so that the score ranged from a minimum of 0 to a maximum of 12 points. Finally, we simplified the risk categorization into 2 levels (likely or unlikely) and not 3 (low, intermediate, and high) to allow a clear-cut decision 
without intermediate group of unclear significance. Similar techniques have been used in previous model simplifications. ${ }^{17-19}$

\section{Outcome Variable}

The primary outcome was any 30-day potentially avoidable readmission, which was identified using an algorithm called SQLape (Striving for Quality Level and Analyzing of Patient Expenses), as it was done in the derivation study. ${ }^{14}$ Basically, this is a validated computerized algorithm based on administrative data and diagnosis codes, and commonly used since more than five years for benchmarking and comparing the different hospitals in Switzerland. ${ }^{20} 21$ Unavoidable readmissions include readmissions involving a new organ system unknown to be affected during the index admission, as well as foreseeable readmissions, which include transplantation, labor and delivery, chemotherapy or radiotherapy, follow-up or rehabilitation treatment, specific surgical procedures, or some specific difficult to cure disorders. On the opposite, readmissions for treatment complications are classified as avoidable. For example, a pregnant woman hospitalized for pneumonia (index admission) and readmitted 2 weeks later for delivery, would have an unavoidable readmission. Conversely, a patient admitted for delivery (index admission), and readmitted later for vaginal bleeding, would have a potentially avoidable readmission.

The secondary outcome was any 30-day readmission.

\section{Statistical Analysis}

Baseline characteristics were presented as median (IQR), mean (SD), or frequency (\%), as appropriate. If a patient was readmitted several times within the 30-day time frame, each 
admission following the first index one was assessed as both an index admission and a readmission. We calculated the simplified HOSPITAL score for each unit of analysis, i.e. for each hospital discharge. Similar to other prediction model simplifications, ${ }^{17-19}$ we categorized the patients in 2 risk groups according to their score points, rather than in 3 groups as in the original study: ${ }^{22}$ unlikely to be readmitted if 0-4 point(s), and likely to be readmitted if 5 points or more. These categories were created for ease of interpretation, roughly corresponding to a risk of potentially avoidable readmission of more than $15 \%$ in the "likely" category. We compared the prevalence of a positive score for each variable of the simplified HOSPITAL score in patients with versus those without readmission using Student t-test.

Three different analyses were used to assess the accuracy of the simplified HOSPITAL score: 1 ) We calculated the Brier score to assess the overall accuracy of the scoring system, i.e. how close the actual rates of readmission were to the predicted ones. A prediction model with a Brier score $<0.25$ is considered useful (the lower, the better). ${ }^{23}{ }^{24} 2$ ) We calculated the C-statistic of the scoring system, which represents the discriminatory power of the score, i.e. the sensitivity and specificity of the model to discriminate between cases and non-cases. ${ }^{25}{ }^{26}$ Results were presented with $95 \% \mathrm{Cl}$. A C-statistic between 0.5 and 1 means that the score is better than random to predict the outcome (the higher the C-statistic, the better the model). 4) We assessed the calibration by fitting a logistic regression model to the data, and comparing the resultant estimates of the predicted readmission risk with the observed rates. ${ }^{24}$ In this model, we included fixed effects at the hospital level to account for variability within the different sites.Furthermore, a robust sandwich variance estimator was used to take into account repeated admissions from a single patient. ${ }^{27}$ 
As a rule of thumb, 10 outcomes are need for each variable tested in a logistic regression model. With around 11,000 outcomes in our cohort population, we will have a large enough population to validate the score that contains 7 variables. ${ }^{28}$

All tests were conducted as two-sided at a 0.05 level of significance. Analyses were performed with SAS Software, version 9.3 (SAS Institute Inc).

\section{RESULTS}

Out of 121,136 discharges from one of the 9 hospitals during the study, 4,701 were excluded because the patients left against medical advice or were transferred to another acute care hospital (Figure 1). Among the 117,065 discharges remaining for analysis, 16,992 (14.5\%) were followed by any readmission within 30 days, and $9.7 \%(n=11,307)$ by a potentially avoidable readmission.

Mean (SD) age of the patients at inclusion was 60.8 (18.2) years and median (IQR) length of stay during the index admission was 4 (3-7) days. Table 2 reports the baseline characteristics of the study population according to the presence or absence of a 30-day potentially avoidable readmission. Each variable of the HOSPITAL score was significantly more often positive (with a $p<0.001$ for each) in patients with a 30-day potentially avoidable readmission, when compared to those without. Overall, the median (IQR) simplified HOSPITAL score was $3(2-5)$ points, with a range from 0 to 12 points. 
Table 2. Baseline characteristics of the study population according to the presence or absence of 30-day potentially avoidable readmission.

\begin{tabular}{|c|c|c|c|}
\hline & $\begin{array}{c}\text { All } \\
\text { discharges } \\
(n=117,065)\end{array}$ & $\begin{array}{l}\text { With 30-day } \\
\text { PAR (n=11,307) }\end{array}$ & $\begin{array}{l}\text { Without 30-day } \\
\text { PAR (n=105,758) }\end{array}$ \\
\hline Age, years & $60.8(18.2)$ & $61.3(18.0)$ & $60.8(18.3)$ \\
\hline Men & $59,667(51.0)$ & $5,988(53.0)$ & $53,679(50.8)$ \\
\hline \multicolumn{4}{|l|}{ Country } \\
\hline Canada & $11,041(9.4)$ & $820(7.3)$ & $10,221(9.7)$ \\
\hline Israel & $17,608(14.0)$ & $1,592(14.1)$ & $16,016(15.1)$ \\
\hline Switzerland & 9,465 (8.1) & $524(4.6)$ & $8,971(8.5)$ \\
\hline USA & $78,921(67.4)$ & $8,371(74.0)$ & $70,550(66.7)$ \\
\hline $\begin{array}{l}\text { Hemoglobin level at discharge } \\
<120 \mathrm{~g} / \mathrm{l}\end{array}$ & $67,386(57.6)$ & $8,005(70.8)$ & $59,381(56,2)$ \\
\hline $\begin{array}{l}\text { Cancer diagnosis or discharge from } \\
\text { an oncology division }\end{array}$ & $24,315(20.8)$ & $2,853(25.2)$ & $21,462(20.3)$ \\
\hline $\begin{array}{l}\text { Sodium level at discharge } \\
<135 \mathrm{mmol} / \mathrm{l}\end{array}$ & $15,558(13.3)$ & $2,038(18.0)$ & $13,520(12.8)$ \\
\hline
\end{tabular}




\begin{tabular}{|l|c|c|c|}
\hline Urgent or emergent index & $88,077(75.2)$ & $9,369(82.9)$ & $78,708(74.4)$ \\
admission (nonelective) & & & \\
\hline Number of hospital Admissions & & $4,826(42.7)$ & $76,196(72.1)$ \\
$0-1$ & $81,022(69.2)$ & $4,464(39.5)$ & $25,109(23.7)$ \\
$2-5$ & $29,573(25.2)$ & $2,017(17.8)$ & $4,453(4.2)$ \\
$\geq 5$ & $6,470(5.5)$ & $6,058(53.6)$ & $45,655(43.2)$ \\
\hline Hospital stay $\geq 5$ days during index & $51,710(4.4)$ & & \\
admission & & & \\
\hline
\end{tabular}

Data are $\mathrm{n}(\%)$ or mean (SD).

Abbreviations: PAR, potentially avoidable readmission; USA, United States of America.

\section{Performance of the simplified HOSPITAL score}

The simplified HOSPITAL score classified $70.4 \%(n=82,383)$ discharges as unlikely, and $29.6 \%$ $(n=34,682)$ as likely to be followed by a 30 -day potentially avoidable readmission (Table $3 a)$. The percentage of discharges followed by a potentially avoidable readmission was $6.4 \%$ in the low-risk category and $17.3 \%$ in the high-risk category (Table 3a). The overall performance was very good, as reflected by a Brier score of 0.08 . Discriminatory power was good also, with a Cstatistic of $0.69(95 \% \mathrm{Cl} 0.68-0.69)$. Figure 2 shows the receiving operating characteristic curves of the simplified HOSPITAL score. The negative predictive value of the simplified HOSPITAL score was $94 \%$, and its specificity $73 \%$. The calibration was excellent with predicted rates matching exactly the observed rates, as shown in Table 3a. When taking any 30-day readmission as outcome, the C-statistic was $0.76(95 \% \mathrm{Cl} 0.76-0.77)$ and the calibration remained excellent (Table 3b). Overall, $29.6 \%$ of the patients were classified as high-risk, and $27.2 \%$ of them had any 30 -day readmission, and $17.3 \%$ had a 30 -day potentially avoidable readmission. 
Table 3a. Observed proportions versus estimated risk of 30-day potentially avoidable readmission with the simplified HOSPITAL score.

\begin{tabular}{|c|c|c|c|c|}
\hline Points & $\begin{array}{c}\text { Risk of 30- } \\
\text { day } \\
\text { readmission }\end{array}$ & $\begin{array}{c}\text { Patients in each } \\
\text { category, } \mathbf{n} \text { (\%) }\end{array}$ & $\begin{array}{c}\text { Observed } \\
\text { proportion with } \\
\text { PAR (\%) }\end{array}$ & $\begin{array}{c}\text { Estimated risk of PAR } \\
\text { using the simplified } \\
\text { HOSPITAL score (\%) }\end{array}$ \\
\hline $0-4$ & unlikely & $82,383(70.4)$ & 6.4 & 6.4 \\
\hline$\geq 5$ & likely & $34,682(29.6)$ & 17.3 & 17.3 \\
\hline
\end{tabular}

Abbreviations: PAR, potentially avoidable readmission.

Table 3b. Observed proportions versus estimated risk of all 30-day readmissions with the simplified HOSPITAL score.

\begin{tabular}{|c|c|c|c|c|}
\hline Points & $\begin{array}{c}\text { Risk of 30- } \\
\text { day } \\
\text { readmission }\end{array}$ & $\begin{array}{c}\text { Patients in each } \\
\text { category, } \mathbf{n}(\%)\end{array}$ & $\begin{array}{c}\text { Observed } \\
\text { proportion of 30- } \\
\text { day readmission (\%) }\end{array}$ & $\begin{array}{c}\text { Estimated risk of 30- } \\
\text { day readmission } \\
\text { using the simplified } \\
\text { HOSPITAL score (\%) }\end{array}$ \\
\hline $0-4$ & unlikely & $82,383(70.4)$ & 9.2 & 9.2 \\
\hline$\geq 5$ & likely & $34,682(29.6)$ & 27.2 & 27.2 \\
\hline
\end{tabular}

\section{DISCUSSION}

In this study including 117,065 medical discharges, we showed that a simplified version of the HOSPITAL score can successfully predict 30-day potentially avoidable, as well as any readmissions. Among the $29.6 \%$ discharges likely to be followed by a 30 -day potentially avoidable readmission, the risk of potentially avoidable readmission according to the score was $17.3 \%$, while the observed proportion was $17.3 \%$, showing excellent calibration. Overall accuracy was very good also, as reflected by a Brier score of 0.08 . With a C-statistic of 0.69 , the 
simplified HOSPITAL score showed similar discriminatory power as the original HOSPITAL score. $^{214}$

The simplified HOSPITAL score offers two advantages in comparison to the original score. First, replacing the variable "discharge from an oncologic division" by "cancer diagnosis or discharge from an oncology division" enables a more extensive propagation of the use of the score in other hospital settings, including those without an oncologic division. Second, removing the variable "any procedure during index admission" probably makes the score more attractive for clinicians, as capturing this variable may be difficult depending on the setting. The simplified HOSPITAL score is therefore even easier to calculate at bedside or automatically calculated in the electronic health record.

In the original derivation and validation studies, patients were classified into 3 categories of risk of potentially avoidable readmission, i.e. low-, intermediate- and high-risk. ${ }^{214}$ Using this 3-levels classification, clinicians may be unsure about how to deal with patients at intermediate risk; therefore, they may aim transition care interventions at high-risk patients only. Doing so may be detrimental for patients classified at intermediate risk but who could benefit of such interventions. A dichotomous classification may also be more convenient in clinical practice, as shown in previous simplification score studies. ${ }^{17-19}$ In this study, we therefore purposely decided to classify the patients into 2 categories of risk only, i.e. unlikely and likely to be readmitted, and consider that this 2-level scheme may be more useful to identify the patients that would most benefit of intensive transition care interventions. However, as for any prediction model, the cutoff chosen (here 5 points or more) might need to be adapted to the setting for better classification.

The HOSPITAL score presents two main advantages. First, it predicts avoidable readmissions, rather than any readmissions, which is of substantial importance, as discharge interventions 
should be aimed at patients that would most likely benefit, rather than for unavoidable readmissions. Second, it can be calculated before discharge, enabling targeted interventions at that time. Although one may argue that an identification of high-risk patients at admission would be more helpful to implement interventions as soon as possible, risk evolves over the course of the hospitalization, and interventions showed to be effective were mostly performed after hospital discharge.

The aim of the HOSPITAL score was to easily identify the patients at high risk of readmission, and the variables included must be seen as good predictors, and not as an exhaustive list of modifiable risk factors. The HOSPITAL score is indeed not including factors that may be seen as very important in the risk of readmission such as socio-economic parameters, follow-up care, or home support. A good score should be easy to use, and predict with good reliability which patients are at high risk. This is what the HOSPITAL score is doing, and its good performance has been now widely validated in nearly 200,000 patients at 16 hospitals, across 5 countries and 3 continents. ${ }^{2-12}$ Because hospital readmission is particularly multifactorial, none can however expect a prediction model for readmission to reach a perfect prediction. Also, the HOSPITAL score identifies a group of patients at high risk, and not the risk at the patient level. There is however no evidence that a risk identification at the patient level is actually more useful than at a high-risk group level.

This study has some limitations. First, we included only medical patients, so that our findings may not be generalizable to surgical populations. Second, we studied the rate of readmission within 30 days after hospital discharge, a cut-off that might always be debatable, but that was chosen because it is the standard used for the Readmission Reduction Program of the Centers for Medicare and Medicaid Services in the United States, as well as in readmission policies of main European countries. ${ }^{29} 30$ Third, because some variables of the score may differ between 
different countries (e.g. the variable "length of stay"), we are not allowed to generalize our findings to any country; however, the validation in 4 countries on 3 continents suggests a large generalizability of the score. Fourth, the algorithm used to differentiate the potentially avoidable from the non-avoidable readmissions may not provide a perfect discrimination. However, no method can argue to have a $100 \%$ sensitivity and specificity to identify the true avoidable readmissions. But the SQlape algorithm has some advantages in comparison to other methods: it has clear criteria and face validity, it is highly reproducible since based mainly on ICD codes, and it allows large database analysis. Also, we ran a sensitivity analysis with any 30day readmission as outcome, that showed an even better discrimination power. Finally, identifying the patients at high risk of readmission with the HOSPITAL score doesn't give any information on which intervention should be performed, but allows to restrict the most promising and complex interventions to the patients who might benefit the most. Intervention studies targeting this population need to be done to prove the clinical impact of the use of the HOSPITAL score.

\section{CONCLUSIONS}

In conclusion, we showed that a simplified version of the HOSPITAL score doesn't decrease its accuracy and clinical utility, but has the potential to widen the settings in which it can be used. 


\section{REFERENCES}

1. Donzé J, Lipsitz S, Bates DW, et al. Causes and patterns of readmissions in patients with common comorbidities: retrospective cohort study. BMJ (Clinical research ed) 2013;347:f7171.

2. Donze JD, Williams MV, Robinson EJ, et al. International Validity of the HOSPITAL Score to Predict 30-Day Potentially Avoidable Hospital Readmissions. JAMA internal medicine 2016;176(4):496-502. doi: 10.1001/jamainternmed.2015.8462 [published Online First: 2016/03/10]

3. Tsugawa $Y$, Jena $A B$, Figueroa JF, et al. Comparison of Hospital Mortality and Readmission Rates for Medicare Patients Treated by Male vs Female Physicians. JAMA internal medicine 2016 doi: 10.1001/jamainternmed.2016.7875 [published Online First: 2016/12/20]

4. Auerbach AD, Kripalani S, Vasilevskis EE, et al. Preventability and Causes of Readmissions in a National Cohort of General Medicine Patients. JAMA internal medicine 2016;176(4):484-93. doi: 10.1001/jamainternmed.2015.7863 [published Online First: 2016/03/10]

5. van Walraven C, Bennett C, Jennings A, et al. Proportion of hospital readmissions deemed avoidable: a systematic review. CMAJ : Canadian Medical Association journal = journal de l'Association medicale canadienne 2011;183(7):E391-402. doi: 10.1503/cmaj.101860 [published Online First: 2011/03/30]

6. Leppin AL, Gionfriddo MR, Kessler M, et al. Preventing 30-day hospital readmissions: a systematic review and meta-analysis of randomized trials. JAMA internal medicine 
2014;174(7):1095-107. doi: 10.1001/jamainternmed.2014.1608 [published Online First: 2014/05/14]

7. Allaudeen N, Schnipper JL, Orav EJ, et al. Inability of providers to predict unplanned readmissions. Journal of general internal medicine 2011;26(7):771-6. doi: 10.1007/s11606-011-1663-3 [published Online First: 2011/03/15]

8. Aubert CE, Folly A, Mancinetti M, et al. Prospective validation and adaptation of the HOSPITAL score to predict high risk of unplanned readmission of medical patients. Swiss medical weekly 2016;146:w14335. doi: 10.4414/smw.2016.14335 [published Online First: 2016/08/09]

9. Burke RE, Schnipper JL, Williams MV, et al. The HOSPITAL Score Predicts Potentially Preventable 30-Day Readmissions in Conditions Targeted by the Hospital Readmissions Reduction Program. Medical care 2016 doi: 10.1097/mlr.0000000000000665 [published Online First: 2016/10/19]

10. Garrison GM, Robelia PM, Pecina JL, et al. Comparing performance of 30-day readmission risk classifiers among hospitalized primary care patients. Journal of evaluation in clinical practice 2016 doi: 10.1111/jep.12656 [published Online First: 2016/10/04]

11. Cooksley T, Nanayakkara PW, Nickel CH, et al. Readmissions of medical patients: an external validation of two existing prediction scores. QJM : monthly journal of the Association of Physicians 2016;109(4):245-8. doi: 10.1093/qjmed/hcv130 [published Online First: 2015/07/15]

12. Robinson R. The HOSPITAL score as a predictor of 30 day readmission in a retrospective study at a university affiliated community hospital. PeerJ 2016;4:e2441. doi: 10.7717/peerj.2441 [published Online First: 2016/09/22] 
13. Collins GS, Reitsma JB, Altman DG, et al. Transparent Reporting of a multivariable prediction model for Individual Prognosis or Diagnosis (TRIPOD): the TRIPOD statement. Annals of internal medicine 2015;162(1):55-63. doi: 10.7326/M14-0697

14. Donzé J, Aujesky D, Williams D, et al. Potentially avoidable 30-day hospital readmissions in medical patients: derivation and validation of a prediction model. JAMA internal medicine 2013;173(8):632-38. doi: 10.1001/jamainternmed.2013.3023

15. World Health Organization. International Classification of Diseases, Ninth Revision (ICD-9). Geneva, Switzerland: World Health Organization; 1977

16. World Health Organization. International Classification of Diseases, Tenth Revision (ICD-10). Geneva, Switzerland: World Health Organization; 1992

17. Klok FA, Mos IC, Nijkeuter M, et al. Simplification of the revised Geneva score for assessing clinical probability of pulmonary embolism. Archives of internal medicine 2008;168(19):2131-6. doi: 10.1001/archinte.168.19.2131 [published Online First: 2008/10/29]

18. Jimenez D, Aujesky D, Moores $L$, et al. Simplification of the pulmonary embolism severity index for prognostication in patients with acute symptomatic pulmonary embolism. Archives of internal medicine 2010;170(15):1383-9. doi: 10.1001/archinternmed.2010.199 [published Online First: 2010/08/11]

19. Gibson NS, Sohne M, Kruip MJ, et al. Further validation and simplification of the Wells clinical decision rule in pulmonary embolism. Thrombosis and haemostasis 2008;99(1):229-34. doi: 10.1160/th07-05-0321 [published Online First: 2008/01/25]

20. Halfon P, Eggli Y, van Melle G, et al. Measuring potentially avoidable hospital readmissions. Journal of clinical epidemiology 2002;55(6):573-87. [published Online First: 2002/06/14] 
21. SQlape. Potentially avoidable readmissions. http://www.sqlape.com. 2013. Accessed online December 18.

22. Halfon P, Eggli Y, Prêtre-Rohrbach I, et al. Validation of the potentially avoidable hospital readmission rate as a routine indicator of the quality of hospital care. Medical care 2006;44(11):972-81. doi: 10.1097/01.mlr.0000228002.43688.c2

23. Khudyakov $P$, Gorfine $M$, Zucker $D$, et al. The impact of covariate measurement error on risk prediction. Statistics in medicine 2015;34(15):2353-67. doi: 10.1002/sim.6498

24. Steyerberg EW, Vickers AJ, Cook NR, et al. Assessing the performance of prediction models: a framework for traditional and novel measures. Epidemiology (Cambridge, Mass) 2010;21(1):128-38. doi: 10.1097/EDE.0b013e3181c30fb2

25. Hanley JA, McNeil BJ. The meaning and use of the area under a receiver operating characteristic (ROC) curve. Radiology 1982;143(1):29-36. doi: 10.1148/radiology.143.1.7063747

26. Pencina MJ, D'Agostino RB. Evaluating Discrimination of Risk Prediction Models: The C Statistic. Jama 2015;314(10):1063-64. doi: 10.1001/jama.2015.11082

27. Zeger SL, Liang KY. Longitudinal data analysis for discrete and continuous outcomes. Biometrics 1986;42(1):121-30. [published Online First: 1986/03/01]

28. Peduzzi P, Concato J, Feinstein AR, et al. Importance of events per independent variable in proportional hazards regression analysis. II. Accuracy and precision of regression estimates. Journal of clinical epidemiology 1995;48(12):1503-10. [published Online First: $1995 / 12 / 01]$

29. Centers for Medicare \& Medicaid Services. Readmissions Reduction Program (HRRP). https://www.cms.gov/medicare/medicare-fee-for-service- 
payment/acuteinpatientpps/readmissions-reduction-program.html. Accessed on June 6, 2016.

30. Kristensen SR, Bech M, Quentin W. A roadmap for comparing readmission policies with application to Denmark, England, Germany and the United States. Health policy (Amsterdam, Netherlands) 2015;119(3):264-73. doi: 10.1016/j.healthpol.2014.12.009 [published Online First: 2014/12/31] 


\section{FIGURE LEGENDS}

Figure 1. Study flow-chart.

Figure 2. Receiving operating characteristic curve of the simplified HOSPITAL score. The Cstatistic was 0.69 (95\%Cl 0.68-0.69). 